\title{
Observation of hydroperoxyethyl formate from the reaction between the methyl Criegee intermediate and formic acid $\dagger$
}

\author{
Carlos Cabezas iD $\ddagger$ and Yasuki Endo* \\ Rapid reactions of carboxylic acids with Criegee intermediates provide efficient gas phase removal \\ processes and are proposed to trigger the formation of new molecular compounds, which are impli- \\ cated in the growth of atmospheric aerosols. The new adducts formed in these reactions are known as \\ hydroperoxide esters and are predicted to be condensable, with lower vapor pressures than the \\ reactants. We report here the direct detection of hydroperoxyethyl formate $\left(\mathrm{HOOCH}\left(\mathrm{CH}_{3}\right) \mathrm{OCHO}\right)$ \\ formed in the gas phase reaction between the methyl substituted Criegee intermediate, $\mathrm{CH}_{3} \mathrm{CHOO}$, and \\ formic acid. Two different isomers of this hydroperoxide ester have been observed using the combi- \\ nation of pulsed Fourier-transform microwave spectroscopy with isotopic substitution experiments and \\ high-level quantum chemical calculations. The identification of hydroperoxyethyl formate as the main \\ nascent product of the $\mathrm{CH}_{3} \mathrm{CHOO}$ and $\mathrm{HCOOH}$ reaction differs from previous results that reported the \\ formation of vinyl hydroperoxide as the reaction product of this reaction.
}

\section{Introduction}

Criegee intermediates (CIs) are carbonyl oxides proposed by Rudolph Criegee, in 1949, as key intermediates in the reaction of ozone with alkenes. These ozonolysis reactions proceed, in a first step, via a 1,3-cycloaddition of ozone across the olefinic bond to form a primary ozonide with a $\mathrm{C}-\mathrm{C}$ single bond, which decomposes, in a second step, into a carbonyl and carbonyl oxide, commonly known as the Criegee intermediate (CI). ${ }^{1,2}$

The atmospheric implications of CIs have been discussed for decades, and now it is known that they play important roles in many aspects of atmospheric chemistry. Nascent CIs, formed vibrationally excited, undergo unimolecular decomposition yielding the hydroxyl radical, $\mathrm{OH}^{3-6}$ On the other hand, the remaining CIs are collisionally stabilized, named stabilized Criegee intermediates (SCIs), but they can still react driven by their zwitteronic character, with themselves or with several important atmospheric species. Thus, SCIs are considered as the main oxidizing agents of atmospheric species such as $\mathrm{SO}_{x}$ and $\mathrm{NO}_{x}$. The reactions of SCIs with water, because of its high concentration in the Earth's atmosphere, is likely to be the

Department of Applied Chemistry, Science Building II, National Chiao Tung University, 1001 Ta-Hsueh Rd., Hsinchu 30010, Taiwan.E-mail: endo@nctu.edu.tw $\dagger$ Electronic supplementary information (ESI) available: Optimized geometries for all the considered species and the measured transition frequencies for the detected ones. See DOI: 10.1039/c9cp05030b

\$ Present address: Instituto de Física Fundamental (IFF-CSIC), Group of Molecular Astrophysics, C/Serrano 121, 28006 Madrid, Spain. dominant sink for SCIs. ${ }^{7,8}$ In addition, reactions of SCIs with organic acids, aldehydes, $\mathrm{HO}_{x}$ and $\mathrm{O}_{3}{ }^{9-11}$ are sources of atmospheric secondary organic aerosols (SOAs). ${ }^{9}$

Because of the CI's short lifetimes, direct experiments to study the CIs reactions have been hampered until recently, and most of the available information about the mechanisms of related reactions has been restricted to indirect experimental evidence or theoretical calculations. However, Taatjes and coworkers $^{12}$ in 2012 introduced a new reaction scheme for CIs involving photolysis of 1,1-diiodo alkanes and a subsequent reaction with $\mathrm{O}_{2}$ stimulating a plethora of kinetic and spectroscopic studies on CIs. Now, the understanding of the Criegee chemistry mainly lies in the determination of the rate coefficients of the reactions with other atmospheric species. Thus, the rate coefficients for the reactions of the simplest $\mathrm{CI}, \mathrm{CH}_{2} \mathrm{OO}$, and the methyl derivative, $\mathrm{CH}_{3} \mathrm{CHOO}$, with $\mathrm{H}_{2} \mathrm{O},{ }^{12-18} \mathrm{NO}_{x}{ }^{12,13,19} \mathrm{SO}_{2}{ }^{12,13}$ or $\mathrm{HCOOH},{ }^{20,21}$ among others, have been determined just in the last decade.

In addition to the kinetic studies, CIs and their reactions with atmospheric species have been investigated from the structural-spectroscopic point of view. These studies are devoted to unveil the distinct conformations that CIs can adopt, as well as the nature of the products derived in their reactions with other atmospheric species. The molecular structures of CIs, such as $\mathrm{CH}_{2} \mathrm{OO}$ and the alkyl-derivatives $\mathrm{CH}_{3} \mathrm{CHOO},\left(\mathrm{CH}_{3}\right)_{2} \mathrm{COO}$ and $\mathrm{C}_{2} \mathrm{H}_{5} \mathrm{CHOO}$, have been investigated using $\mathrm{UV}^{22-24} \mathrm{IR}^{25,26}$ and rotational spectroscopies. ${ }^{27-33}$ Larger $\mathrm{CIs}\left(\mathrm{CH}_{3}\right)_{2} \mathrm{CHCHOO}{ }^{34}$ $\mathrm{C}_{2} \mathrm{H}_{5}\left(\mathrm{CH}_{3}\right) \mathrm{COO}^{35,36}$ and $\mathrm{C}_{3} \mathrm{H}_{7} \mathrm{CHOO}^{37}$ and halogen-derivatives 
like $\mathrm{ClCHOO}^{38}$ have only been investigated in our lab using rotational spectroscopy, which is very well suited for this kind of study in the gas phase due to its high resolution and sensitivity. These studies describe the detection and identification of, for example, up to four different conformers for $\left(\mathrm{CH}_{3}\right)_{2} \mathrm{CHCHOO}$ or $\mathrm{C}_{3} \mathrm{H}_{7} \mathrm{CHOO}$. Knowledge of the conformational preferences of CIs is crucial since it is known that their self-reactivity and their reactivity with other atmospheric species are conformation dependent. Rotational spectroscopy has also been applied to investigate the reactions of CIs, $\mathrm{CH}_{2} \mathrm{OO}$ and $\mathrm{CH}_{3} \mathrm{CHOO}$, towards $\mathrm{H}_{2} \mathrm{O}$, ${ }^{39-41}$ $\mathrm{HCl}^{42,43}$ and $\mathrm{HCOOH}^{44}$ In these studies, the pre-reactive complexes between $\mathrm{CH}_{2} \mathrm{OO}$ and $\mathrm{CH}_{3} \mathrm{CHOO}$ with $\mathrm{H}_{2} \mathrm{O}$ were spectroscopically characterized in addition to the corresponding hydroperoxide adducts resulting from the insertion of $\mathrm{H}_{2} \mathrm{O}, \mathrm{HCl}$ and $\mathrm{HCOOH}$ into the CIs.

In this paper, we report the detection of hydroperoxyethyl formate ( $\left.\mathrm{HPEF}, \mathrm{HOOCH}\left(\mathrm{CH}_{3}\right) \mathrm{OCHO}\right)$ as the nascent product from the reaction between the methyl $\mathrm{CI}, \mathrm{CH}_{3} \mathrm{CHOO}$, and formic acid, $\mathrm{HCOOH}$. Prior to the experimental studies, the reaction has been explored using quantum-chemical computations to elucidate the reaction mechanism. Our calculations provide detailed information on the formation mechanisms of HPEF, since, to our knowledge, there are no previous data available in the literature. Then, supported by the $a b$ initio calculations and using Fourier transform microwave (FTMW) spectroscopy, we have characterized the products resulting from the $\mathrm{CH}_{3} \mathrm{CHOO}+\mathrm{HCOOH}$ reaction. Welz et al. ${ }^{20}$ in 2014 employed multiplexed photoionization mass spectrometry (MPIMS) to measure the rate coefficients for this reaction and they found very large values of 2.5 and $5 \times 10^{-10} \mathrm{~cm}^{3} \mathrm{molec}^{-1} \mathrm{~s}^{-1}$ for $s y n$ - and anti- $\mathrm{CH}_{3} \mathrm{CHOO}$, respectively. These values indicate that the rapid reactions of CIs with $\mathrm{HCOOH}$ can make a substantial contribution to global atmospheric chemistry. However, they could not find any evidence for the products resulting from this reaction. In 2015, Liu et al. ${ }^{45}$ reported the detection, using VUV photoionization, of vinyl hydroperoxide (VHP) formed via formic acid-catalyzed tautomerization of
$\mathrm{CH}_{3}$ CHOO. In that reaction, a doubly hydrogen bonded interaction between $s y n-\mathrm{CH}_{3} \mathrm{CHOO}$ and $\mathrm{HCOOH}$ facilitates efficient hydrogen transfer through a double hydrogen shift. Despite being kinetically and thermodynamically more stable than VHP, HPEF was not observed by Liu et al. ${ }^{45}$ for the reaction of $s y n-\mathrm{CH}_{3} \mathrm{CHOO}$ with formic acid. Hence, the results presented here help to elucidate the formation mechanism of hydroperoxides resulting from CIs and provide targets for chemical modeling studies of these important atmospheric species.

\section{Ab initio calculations}

Quantum chemical calculations, using the Molpro 2018.1 $a b$ initio program package, ${ }^{46}$ were carried out to examine the potential energy surface for the $\mathrm{CH}_{3} \mathrm{CHOO}+\mathrm{HCOOH}$ reaction. All the stationary points were fully optimized using the coupled cluster singles and doubles correlated (CCSD) method along with Dunning's augmented correlation consistent polarized valence triple- $\zeta$ (aug-cc-pVTZ) basis set. ${ }^{47}$ Fig. 1 depicts the potential energy surface along the reaction coordinate for the system studied in this work. The stationary point of the syn- $\mathrm{CH}_{3} \mathrm{CHOO}$ conformer and $\mathrm{HCOOH}$ separated from each other was assumed to be the energy zero.

The addition of $\mathrm{CH}_{3} \mathrm{CHOO}$ (either syn and anti) to formic acid yields the insertion product HPEF. This reaction can proceed through different pathways to lead to two different conformers of HPEF from syn- and anti- $\mathrm{CH}_{3} \mathrm{CHOO}$, respectively. In total, four conformers, differing in the disposition of the $-\mathrm{C}(\mathrm{O}) \mathrm{H}$ group over the $\mathrm{OOH}$ and $\mathrm{CH}_{3}$ moieties, can be formed, as shown in Fig. 2. $\mathrm{CH}_{3} \mathrm{CHOO}$ may attack the $\mathrm{O}-\mathrm{H}$ group in a 1,2-insertion reaction through the formation of pre-reactive complexes that lie at 16.74 and $17.53 \mathrm{kcal} \mathrm{mol}^{-1}$ below the reagents, for the $\mathrm{syn}-\mathrm{CH}_{3} \mathrm{CHOO}$ and anti- $\mathrm{CH}_{3} \mathrm{CHOO}$, respectively. The transition states between the complexes, structures shown in Fig. 3, and the products, syn-HPEF-I and anti-HPEF-II, lie below the reagents by 15.49 and $15.57 \mathrm{kcal} \mathrm{mol}^{-1}$. In order to verify that the pre-reactive complexes

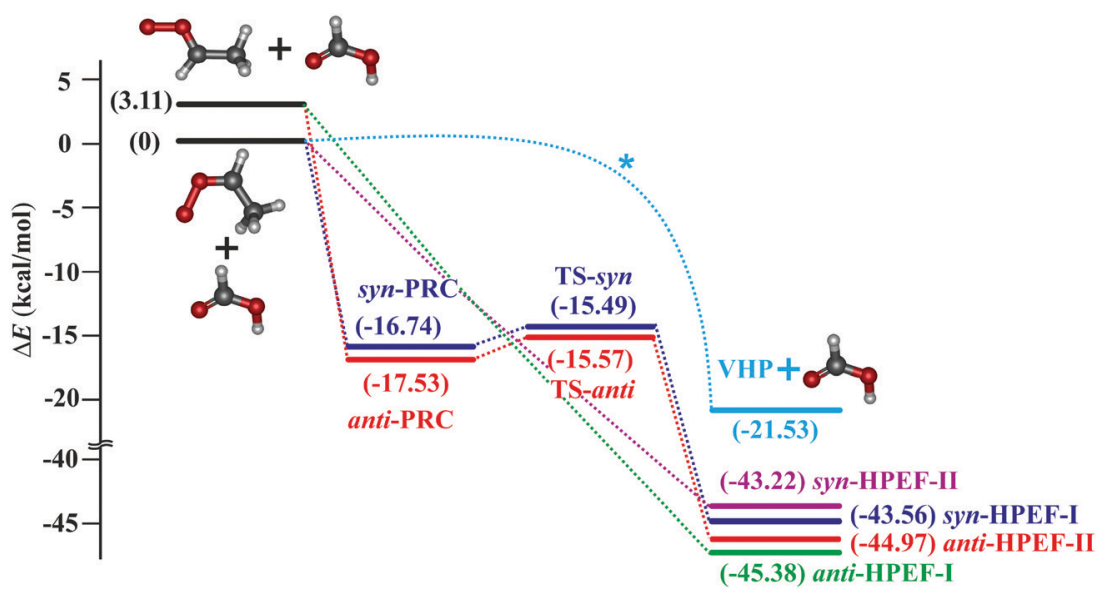

Fig. 1 Energy diagram of the $\mathrm{CH}_{3} \mathrm{CHOO}+$ formic acid reaction. All the calculations were performed at the CCSD/aug-cc-pVTZ level of theory. Total energies relative to that of separated molecules are given in parentheses (in $\mathrm{kcal} \mathrm{mol}^{-1}$ ). The reaction pathway marked with the asterisk has not been calculated in this work, see text for details. 


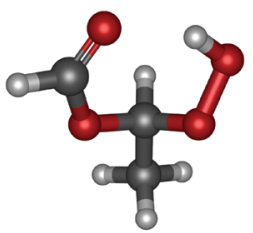

anti-HPEF-I

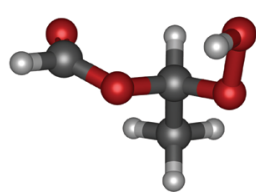

anti-HPEF-II

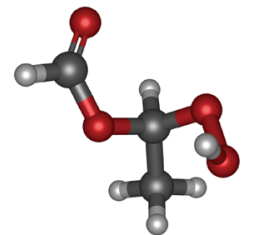

syn-HPEF-I

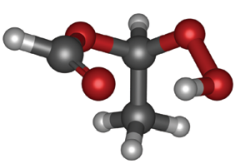

syn-HPEF-II

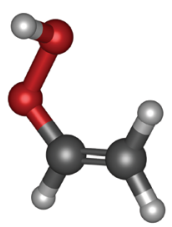

VHP

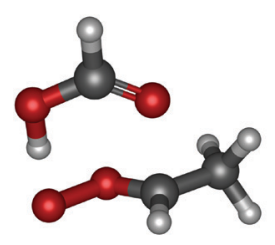

anti-PRC

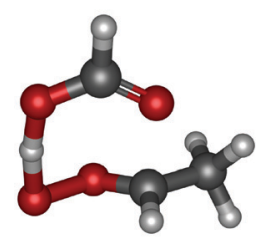

TS-anti

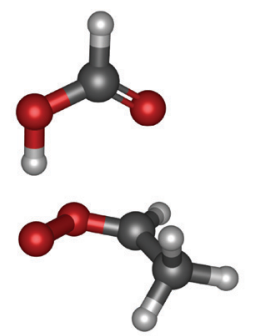

syn-PRC

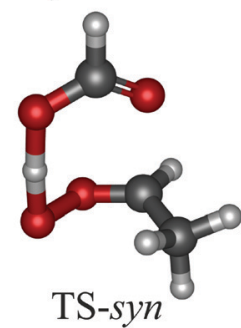

Fig. 3 Pre-reactive complex and transition state structures obtained from $a b$ initio calculations at the CCSD/aug-cc-pVTZ level of theory.

(PRCs) and the insertion products were connected through these transition states, we carried out intrinsic reaction coordinate (IRC) calculations at the CCSD/aug-cc-pVTZ level of theory. The 1,4-insertion reaction, where $\mathrm{CH}_{3} \mathrm{CHOO}$ attacks the $\mathrm{O}=\mathrm{C}-\mathrm{OH}$ moiety, was also explored starting from multiple approaching trajectories between the two reactants, but only the syn- and
anti-PRCs were found. Using IRC calculations, we discard that these PRCs were connected with syn-HPEF-II and anti-HPEF-I conformers via TS-syn and TS-anti, respectively, and no other TS could be located. Hence, the 1,4-insertion reaction to form the conformers syn-HPEF-II and anti-HPEF-I seems to be a barrierless pathway. A similar behavior was found for the reaction of simplest CI, $\mathrm{CH}_{2} \mathrm{OO}$, and $\mathrm{HCOOH}{ }^{44,48,49}$

A different pathway for the $s y n-\mathrm{CH}_{3} \mathrm{CHOO}+\mathrm{HCOOH}$ reaction lead to vinyl hydroperoxide $\left(\mathrm{CH}_{2}=\mathrm{CHOOH}\right)$, VHP. Liu et al. ${ }^{45}$ and Kumar et al. ${ }^{50}$ proposed that $s y n-\mathrm{CH}_{3} \mathrm{CHOO}$ can produce VHP in an exothermic process with formic acid facilitating the hydrogen transfer through a double hydrogen shift. The detailed calculations for this tautomerization pathway have not been performed in this work since they have already been deeply scrutinized by Kumar et al. ${ }^{50}$ Those calculations at the $\operatorname{CCSD}(\mathrm{T}) /$ aug-cc-pVTZ//M06-2X/aug-cc-pVTZ level of theory indicate that the $\mathrm{H}$-transfer passes through two reaction intermediates and a transition state to finally generate VHP and formic acid. In this work, we have only calculated the energy of the final product resulting from this channel and we found that this stationary point is lying below the reactants by $21.5 \mathrm{kcal} \mathrm{mol}^{-1}$. The results are shown in Fig. 1 and 2.

According to our calculations, the four conformations of HPEF together with VHP are potentially observable, by FTMW spectroscopy, products of the $\mathrm{CH}_{3} \mathrm{CHOO}+\mathrm{HCOOH}$ reaction since their formation reactions are all exothermic. Accurate molecular rotational constants and electric dipole moment components are necessary to predict the rotational transition frequencies of these species. The values obtained for these parameters at the CCSD/aug-cc-pVTZ level of theory are summarized in Table 1.

\section{Experimental}

The products from the $\mathrm{CH}_{3} \mathrm{CHOO}+\mathrm{HCOOH}$ reaction were generated in a supersonic jet by an electric discharge of a gas mixture of diiodoethane $/ \mathrm{O}_{2} / \mathrm{HCOOH}$ diluted in Ar. A premix gas of $0.3 \%$ of $\mathrm{HCOOH}, 2 \% \mathrm{O}_{2}$ and $97.7 \%$ Ar with a stagnation pressure of 1.5 atm was passed through a liquid container filled with diiodoethane in order to introduce the precursor with sufficient partial pressure. Then, the gas mixture was flowed through a pulsed-solenoid valve that is accommodated in the backside of one of the cavity mirrors and aligned parallel to the

Table 1 Rotational constants and electric dipole moment components for the four HPEF conformers and VHP calculated at the CCSD/aug-cc-pVTZ level of theory

\begin{tabular}{|c|c|c|c|c|c|}
\hline & anti-HPEF-I & anti-HPEF-II & syn-HPEF-I & syn-HPEF-II & VHP \\
\hline$B / \mathrm{MHz}$ & 2616 & 1953 & 1908 & 2618 & 6721 \\
\hline$C / \mathrm{MHz}$ & 1617 & 1529 & 1634 & 2036 & 5017 \\
\hline$\left|\mu_{\mathrm{b}}\right| / \mathrm{D}$ & 3.02 & 0.23 & 1.20 & 2.73 & 0.20 \\
\hline$\left|\mu_{\mathrm{c}}\right| / \mathrm{D}$ & 0.74 & 1.80 & 2.33 & 0.57 & 1.42 \\
\hline$\Delta E / \mathrm{kcal} \mathrm{mol}^{-1}$ & $0^{b}$ & 0.41 & 1.82 & 2.16 & 23.85 \\
\hline
\end{tabular}

${ }^{a} 1 \mathrm{D} \approx 3.3356 \times 10^{-30} \mathrm{C} \mathrm{m} .{ }^{b}$ Relative energy to that of anti-HPEF-I species. 
optical axis of the resonator. A pulsed voltage of $1.0 \mathrm{kV}$ with $450 \mu$ s of duration was applied between electrodes attached at the exit of the nozzle ${ }^{51,52}$ to induce an electric discharge, synchronized with the gas expansion, which triggers the chemical reactions in the precursor mixture. The resulting products generated in the discharge are supersonically expanded and rapidly cooled to a rotational temperature of $\sim 2 \mathrm{~K}$ and probed by FTMW spectroscopy using a Balle-Flygare type Fourier-transform cavity microwave spectrometer, ${ }^{53}$ previously described in the literature. ${ }^{38}$ All the measurements were carried out in the 10-18 GHz frequency region with an estimated accuracy better than $3 \mathrm{kHz}$, and the resolution better than $5 \mathrm{kHz}$.

Initial measurements were done using normal formic acid, and further experiments were carried out with the monodeuterated species of formic acid, DCOOH (purchased from Sigma). In the latter case, the gas mixture contained $0.3 \%$ of DCOOH, $2 \% \mathrm{O}_{2}$ and $97.7 \% \mathrm{Ar}$.

\section{Results}

As mentioned before, the target species to be found in the rotational spectra are the four conformations of HPEF together with VHP, whose molecular structures are shown in Fig. 2. Prior to the search for these species, we checked the production of both syn- and anti- $\mathrm{CH}_{3} \mathrm{CHOO}$ in the discharged jet using the diiodoethane $/ \mathrm{O}_{2} / \mathrm{HCOOH} / \mathrm{Ar}$ gas mixture. Strong signals were observed at $12362.64 \mathrm{MHz}^{30}$ and $17202.53 \mathrm{MHz}^{31}$ for syn- and anti- $\mathrm{CH}_{3} \mathrm{CHOO}$, respectively, confirming an efficient $\mathrm{CH}_{3} \mathrm{CHOO}$ generation process. The yield of $s y n-\mathrm{CH}_{3} \mathrm{CHOO}$ was estimated to be about five times that of anti- $\mathrm{CH}_{3} \mathrm{CHOO}$, which agrees well with previous works. ${ }^{30,31,41,43}$

At first, experiments were conducted to find rotational transitions of anti-HPEF conformers since they both are predicted as the most stables species. In the initial scans carried out between 10.5 and $14.5 \mathrm{GHz}$, we observed strong signals that appeared close to the predicted rotational frequencies for the anti-HPEF-I conformer. Those lines, without any fine or hyperfine splitting beyond the instrumental Doppler splitting as shown in Fig. 4, were identified as b-type rotational transitions of the conformer anti-HPEF-I. In addition, weaker a- and c-type rotational transitions were measured for this species resulting in a total of 28 observed lines. The relative line intensities are in agreement with the predicted electric dipole moment components for anti-HPEF-I, shown in Table 1. All the observed rotational frequencies, a complete list is available in the ESI, $\dagger$ were included in a least-squares fit using Watson's A-reduced Hamiltonian ${ }^{54}$ and the analysis rendered the experimental rotational constants listed in Table 2 .

Several lines remained unassigned in the scanned regions after the identification of those for the anti-HPEF-I conformer. We tried to ascribe them to the anti-HPEF-II conformer, but after many trials and subsequent measurements, we were not able to obtain a reasonable fit for this conformer. Since the lines are moderately intense, we considered the syn-HPEF conformers as possible carriers of these rotational transitions.

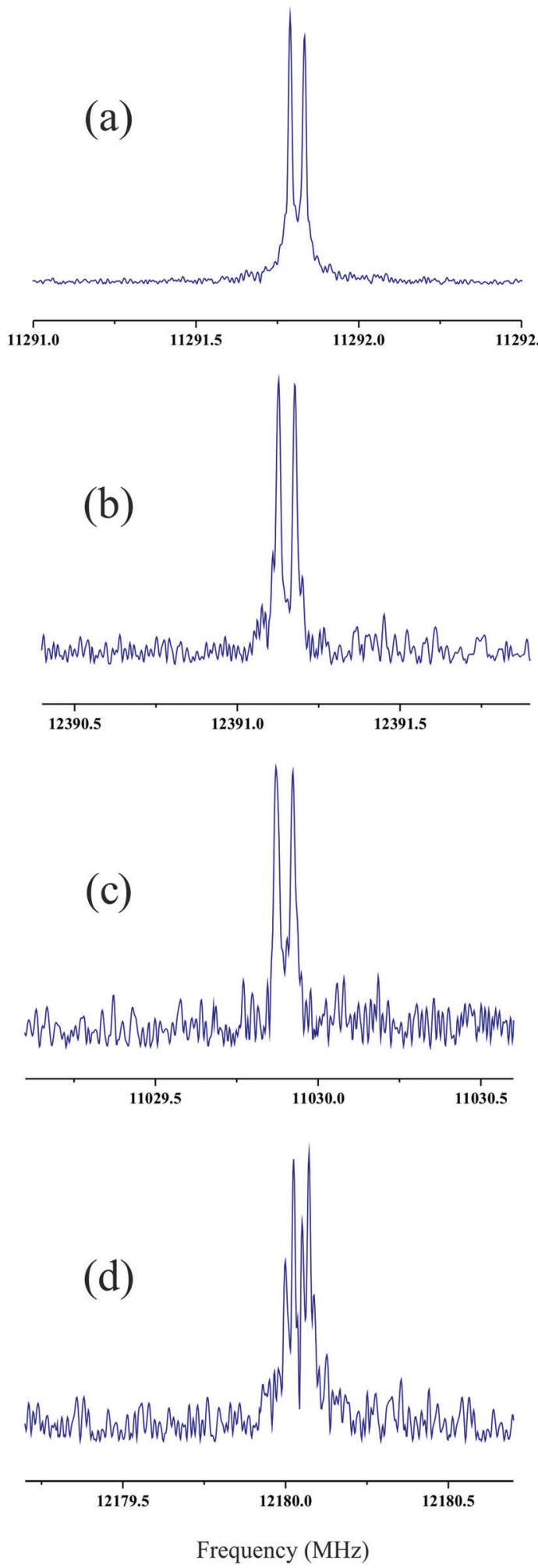

Fig. 4 The FTMW spectra of the $3_{13}-2_{02}$ rotational transitions for (a) antiHPEF-I, (b) syn-HPEF-I, (c) anti-d-HPEF-I and (d) syn-d-HPEF-I species formed in the supersonic expansion. The spectra were achieved by 100-shots of accumulation. Each of the rotational transitions is split into two Doppler components because the direction of the supersonic jet expansion is parallel to the standing wavein the Fabry-Pérot cavity of the spectrometer. In case of the spectrum (d), hyperfine components are observed due to the hyperfine effects of the deuterium nucleus (see text). 
The observed transitions were confirmed to appear very close to the predicted b- and c-type transitions of the syn-HPEF-I conformer, and they were finally ascribed to this conformer, for which a total of 19 rotational transitions, including very weak a-type lines, were observed. The experimental rotational parameters determined for the syn-HPEF-I conformer are shown in Table 2.

Considering the calculated relative energies for the antiHPEF-II and syn-HPEF-II conformers and the line intensity for the anti-HPEF-I and syn-HPEF-I species, the former species should be observed in our rotational spectrum. Hence, we carefully scanned the predicted frequency regions for the rotational transitions of the anti-HPEF-II and syn-HPEF-II species. No lines attributable to them were finally found. Taking into account the $a b$ initio calculations, the formation of VHP is also exothermic and it could be observed under our experimental conditions as well. We also scanned the predicted rotational frequencies for VHP carefully, but no signals ascribable to VHP were found.

Experiments using the deuterated species of formic acid, $\mathrm{DCOOH}$, were carried out to track the passage of the hydrogen atoms from formic acid to HPEF within the reactions. Thus, we observed the monodeuterated species, $\operatorname{HOOCH}\left(\mathrm{CH}_{3}\right) \mathrm{OCDO}$ (d-HPEF) for both anti-HPEF-I and syn-HPEF-I. The presence of the deuterium nucleus, with the nuclear quadrupole moment different from zero $(I=1)$, produces the hyperfine effects on the rotational transition of d-HPEF species due to the interaction of the quadrupole moment with the electric field gradient at the deuterium nucleus. ${ }^{55}$ The hyperfine effects split each rotational transition into several component lines as can be seen in Fig. 4. For syn-d-HPEF-I, the hyperfine splittings are large enough to be resolved and measured with our experimental technique, allowing the experimental determination of the nuclear quadrupole coupling constants in addition to the rotational constants, as shown in Table 2. They were determined by fitting the transition frequencies using Watson's A-reduced Hamiltonian for asymmetric top molecules, with the following form: $H=H_{\mathrm{R}}+H_{\mathrm{Q}}$ where $H_{\mathrm{R}}$ contains rotational and centrifugal distortion parameters while $H_{\mathrm{Q}}$ describes the quadrupole coupling effect. In contrast, the hyperfine splittings for the anti-d-HPEF-I conformer are smaller than those for syn-d-HPEF-I and could not be resolved, resulting in small line broadenings in all the rotational transitions for the anti-dHPEF-I conformer, as it can be seen in Fig. 4. Hence, for this species, only the rotational constants could be derived as shown in Table 2.

\section{Discussion}

The molecular constants for the two identified species of HPEF are provided in Table 2, while the predicted values for the four plausible conformers of HPEF are provided in Table 1. The identifications of both the anti-HPEF-I and syn-HPEF-I conformers have been carried out by comparing their experimental and theoretical values, with very good agreement, where the maximum relative deviations were smaller than $1.0 \%$. At first sight, it seems that the experimental rotational constants for anti-HPEF-I and syn-HPEF-I could also be compatible with those predicted for $s y n$-HPEF-II and anti-HPEF-II, respectively. However, differences between the experimental and theoretical values in such cases are much larger, with relative deviations ranging from 5 to $27 \%$, as seen in Table S1 of the ESI. $\dagger$ The identification of the two observed species as anti-HPEF-I and syn-HPEF-I was finally confirmed by the observation of their monodeuterated isotopic species. As can be seen in Table S1 (ESI $\dagger$ ), the experimental rotational constants for the monodeuterated species agree perfectly with those corrected using the theoretical/experimental factor derived from the parent species. The latter is the most common method used to predict the expected rotational constants for the isotopic species of a given molecule once the rotational constants for its parent species are known. The hypothetical identification of the observed species as syn-HPEF-II and anti-HPEF-II using the data for the monodeuterated species is unlikely due to the disagreement of the expected values for the rotational constants obtained using the correction factors of the parent species shown in Table S1 of the ESI. $\dagger$

The present results can be compared to those reported in a previous work, ${ }^{44}$ where we reported the detection of

Table 2 Experimental rotational parameters for the observed species of HPEF and their isotopologues

\begin{tabular}{|c|c|c|c|c|}
\hline & anti-HPEF-I & anti-d-HPEF-I & syn-HPEF-I & syn-d-HPEF-I \\
\hline $\mathrm{A} / \mathrm{MHz}$ & $3557.66914(11)^{b}$ & $3475.24529(18)$ & $4422.36894(22)$ & $4343.7883(21)$ \\
\hline$B / \mathrm{MHz}$ & $2592.9501(32)$ & $2534.29754(21)$ & $1889.58465(10)$ & $1836.7038(42)$ \\
\hline$C / \mathrm{MHz}$ & $1601.9461(21)$ & $1564.7546(41)$ & $1619.18028(45)$ & $1590.5154(12)$ \\
\hline$\Delta_{J} / \mathrm{kHz}$ & $0.3760(12)$ & {$[0.3760]^{d}$} & $0.13496(17)$ & {$[0.13496]$} \\
\hline$\Delta_{J K} / \mathrm{kHz}$ & $0.3945(41)$ & {$[0.3945]$} & $0.9975(21)$ & {$[0.9975]$} \\
\hline$\Delta_{K} / \mathrm{kHz}$ & $0.926(14)$ & [0.926] & $0.6553(74)$ & [0.6553] \\
\hline$\delta_{J} / \mathrm{kHz}$ & $0.127(72)$ & {$[0.127]$} & $0.3944(32)$ & {$[0.3944]$} \\
\hline$\delta_{K} / \mathrm{kHz}$ & $0.5629(12)$ & {$[0.5629]$} & $0.355(17)$ & {$[0.355]$} \\
\hline$\chi_{\mathrm{aa}} / \mathrm{MHz}$ & - & - & - & $-0.19165(52)$ \\
\hline$\gamma_{\text {bb }} / \mathrm{MHz}$ & - & - & - & $0.08014(12)$ \\
\hline & $28^{c}$ & $9^{c}$ & $19^{c}$ & $37^{e}$ \\
\hline$\sigma^{a} / \mathrm{kHz}$ & 0.6 & 0.3 & 0.7 & 4.7 \\
\hline
\end{tabular}

${ }^{a}$ Standard deviation of the fit. ${ }^{b}$ Values in parentheses denote $1 \sigma$ errors, applied to the last digit. ${ }^{c}$ Number of pure rotational transitions included in the fit. ${ }^{d}$ Values in brackets refer to values fixed to those obtained for the parent species. ${ }^{e}$ Number of hyperfine components included in the fit. 
hydroperoxymethyl formate as the main reaction product from the $\mathrm{CH}_{2} \mathrm{OO}+\mathrm{HCOOH}$ reaction. Two different conformations formed through independent insertion mechanisms, 1,2- and 1,4-insertions, with an energy difference of about $0.57 \mathrm{kcal} \mathrm{mol}^{-1}$ were identified. In the present study, the energy difference between the syn and the anti conformations of HPEF is 0.34 and $0.41 \mathrm{kcal} \mathrm{mol}^{-1}$, respectively, but only the lower energy species of each pair has been observed, syn-HPEF-I and anti-HPEF-I. The explanation of this fact is intricate since $s y n$-HPEF-I and anti-HPEF-I are formed through different insertion mechanisms, 1,2 in the case of the syn-HPEF-I and 1,4 in the case of anti-HPEF-I. Hence, anti-HPEF-II and syn-HPEF-II formed by 1,2 and 1,4 mechanisms, respectively, should be observed as well, considering the energetic data from Fig. 1. On the other hand, in spite of syn-HPEF-I and anti-HPEF-I being the most exothermic products for each of the reaction channels of syn- and anti$\mathrm{CH}_{3} \mathrm{CHOO}$, the difference in the energies of the HPEF-I and HPEF-II products is tiny, compared with the overall exothermicity. Therefore, a thermodynamic explanation for our spectral observations can result in ambiguity. We can conclude, then, that both kinetic and thermodynamical factors drive the formation of specific conformers of hydroperoxymethyl formate in the $\mathrm{CH}_{3} \mathrm{CHOO}+\mathrm{HCOOH}$ reaction.

On the other hand, since our experiments are carried out in a supersonic expansion, we should consider that conformational relaxation processes may occur between the contemplated molecular species. These interconversion processes take place in the early stages of the supersonic expansion by collisions between the different conformers and the buffer gas molecules. Ruoff et al. ${ }^{56}$ have deduced that collisional removal of a higher energy conformer is possible when the isomerization barrier is around $400-500 \mathrm{~cm}^{-1}$ and $\mathrm{Ar}$ is used as a carrier gas. In a previous investigation, we reported conformational interconversion between the anti conformers of $\mathrm{CI}\left(\mathrm{CH}_{3}\right)_{2} \mathrm{CHCHOO}^{34}$ through a barrier height of $479 \mathrm{~cm}^{-1}$. As can be seen in Fig. 2, the molecular structures of the anti-HPEF and syn-HPEF conformers differ exclusively in the relative disposition of the $-\mathrm{C}(\mathrm{O}) \mathrm{H}$ group over the $\mathrm{OOH}$ moiety or in different values of the dihedral angle $\angle$ OCOC. To gain some insight into a plausible interconversion between conformers, we have calculated the potential energy profile for anti-HPEF-II $\rightarrow$ anti-HPEF-I and syn-HPEF-II $\rightarrow$ syn-HPEF-I interconversions by scanning the dihedral angle $\angle$ OCOC. For the anti conformers, an energy barrier around $593 \mathrm{~cm}^{-1}$ has been obtained, which is not high enough to preclude the interconversion between the anti-HPEF-II and anti-HPEF-I conformers. This could be another reason for the nonobservation of the conformer, anti-HPEF-II, which could convert into anti-HPEF-I in the early stages of the supersonic expansion. In contrast, the barrier height between the syn-HPEF-II and syn-HPEF-I conformers is much higher $\left(932 \mathrm{~cm}^{-1}\right)$ than that calculated for the anti conformers, and a conformational interconversion cannot be considered for syn conformers.

Our present observation of HPEF indicates that this hydroperoxide ester is the main nascent product from the $\mathrm{CH}_{3} \mathrm{CHOO}+$ $\mathrm{HCOOH}$ reaction. This result is confirmed by the following experimental evidences: (i) HPEF rotational transitions disappear in absence of electric discharge, and (ii) the lines disappear when any of the reactants (diiodoethane, $\mathrm{O}_{2}$ or $\mathrm{HCOOH}$ ) is removed from the gas mixture. Moreover, the formation mechanism of $\mathrm{HPEF}$ in the reaction from $\mathrm{CH}_{3} \mathrm{CHOO}+\mathrm{HCOOH}$ can be elucidated using the experimental data obtained when the deuterated species of formic acid is used. As mentioned before, the monodeuterated species $\mathrm{HOOCH}\left(\mathrm{CH}_{3}\right) \mathrm{OCDO}$ (d-HPEF) were detected when using $\mathrm{DCOOH}$. This shows that one of the terminal $\mathrm{H}$ atoms of HPEF is transferred from the formic acid molecule proving that the HPEF molecule observed in our experiment can just be produced within the reaction of $\mathrm{CH}_{3} \mathrm{CHOO}$ with $\mathrm{HCOOH}$. A similar reaction mechanism has been shown before for the $\mathrm{CH}_{2} \mathrm{OO}+\mathrm{HCOOH}$ reaction, ${ }^{44}$ where hydroperoxymethyl formate was identified as the main reaction product.

Previously, Liu et al. ${ }^{45}$ investigated the reaction between $\mathrm{CH}_{3} \mathrm{CHOO}$ and $\mathrm{HCOOH}$ using VUV photoionization at $118 \mathrm{~nm}$ under collision-free conditions. The CIs and the resultant products were cooled in a supersonic expansion and detected using a time-of-flight mass spectrometer. Although the experimental conditions used by Liu et al. ${ }^{45}$ bear some similarity with those used in the present work, the final results are completely different. Liu et al. ${ }^{45}$ only observed vinyl hydroperoxide (VHP) formed via formic acid-catalyzed tautomerization of $s y n-\mathrm{CH}_{3} \mathrm{CHOO}$ and reported no experimental evidences for the formation of HPEF. In sharp contrast, our experimental results point to HPEF as the only reaction product from the $\mathrm{CH}_{3} \mathrm{CHOO}+\mathrm{HCOOH}$ reaction, which can be reasonably justified from the thermodynamical point of view. A plausible explanation for the lack of observation of the insertion product is that HPEF undergoes fragmentation upon ionization at $10.5 \mathrm{eV}$, and therefore was not observed on the HPEF parent mass channel. ${ }^{45}$ In addition, differences in the CI's generation method exist between both the experiments, UV photolysis in Liu's experiment and electric discharges in this work. This fact can as well affect the non observation of VHP under our experimental conditions.

Taking into account the observed spectral intensities of several rotational transitions, the relative abundances of the observed two HPEF species in the supersonic expansion can be estimated. The intensity of the rotational transition is proportional to the number density of each conformer and the value of the corresponding component of the dipole moment, $N_{i} \cdot \mu_{i}$. Considering the predicted values of the dipole moment components, the relative abundances of HPEF isomers have been found to be: anti-HPEF-I : syn-HPEF-I $=1.3: 1$. This is in agreement with the theoretical calculations that predict the anti-HPEF-I conformer as the most stable isomer from the kinetic and thermodynamical point of view. Under our experimental conditions, anti- $\mathrm{CH}_{3} \mathrm{CHOO}$ is about five times less abundant than syn- $\mathrm{CH}_{3} \mathrm{CHOO} .{ }^{30,31,41,43}$ According to the present observation, we can infer that the anti- $\mathrm{CH}_{3} \mathrm{CHOO}$ species reacts with $\mathrm{HCOOH}$ much faster than $s y n-\mathrm{CH}_{3} \mathrm{CHOO}$ does, as pointed out by Welz et al. ${ }^{20}$ In addition, we can estimate the upper limit for VHP taking into account the signal-to-noise ratio for HPEF and the predicted dipole moment components. We found that the abundance of VHP under our experimental conditions is at least 21 times smaller than that of HPEF. 


\section{Conclusions}

The work presented here constitutes the first experimental evidence that hydroperoxyethyl formate ( $\left.\mathrm{HPEF}, \mathrm{HOOCH}\left(\mathrm{CH}_{3}\right) \mathrm{OCHO}\right)$ represents the primary nascent product from the atmospheric reaction between the methyl Criegee intermediate, $\mathrm{CH}_{3} \mathrm{CHOO}$, and carboxylic acid, $\mathrm{HCOOH}$. Using high resolution rotational spectroscopy combined with electric discharges, we have probed the mentioned gas phase reaction, reporting spectroscopic evidence, with the help of high-level structural calculations, of HPEF as the dominant reaction product, which eluded direct detection in previous experiments.

Our results differ from those reported before by Liu et al., who observed the formation of vinyl hydroperoxide as the reaction product between $\mathrm{CH}_{3} \mathrm{CHOO}$ and $\mathrm{HCOOH}$. Different experimental conditions used by Liu et al. and those employed in the present work can be the origin of these contrasting results. However, both conclusions are compatible and help to understand the formation of atmospheric condensable products in gas phase oxidation reactions and shed light on the growth mechanisms of secondary organic aerosols.

\section{Conflicts of interest}

There are no conflicts to declare.

\section{Acknowledgements}

This research was supported by the Ministry of Science and Technology of Taiwan under Grant Nos MOST 104-2113-M-009-020 (Y. E.) and MOST 105-2811-M-009-026 and 106-2811-M-009023 (C. C.).

\section{References}

1 R. Criegee and G. Wenner, Die ozonisierung des 9,10oktalins, Justus Liebigs Ann. Chem., 1949, 564, 9.

2 R. Criegee, Mechanism of ozonolysis, Angew. Chem., Int. Ed. Engl., 1975, 14, 745.

3 J. H. Kroll, J. S. Clarke, N. M. Donahue, J. G. Anderson and K. L. Demerjian, Mechanism of $\mathrm{OH}_{x}$ formation in the gasphase ozone-alkene reaction. 1. Direct, pressure-dependent measurements of prompt oh yields, J. Phys. Chem. A, 2001, 105, 1554-1560.

4 J. H. Kroll, S. R. Sahay, J. G. Anderson, K. L. Demerjian and N. M. Donahue, Mechanism of $\mathrm{HO}_{x}$ formation in the gas-phase ozone-alkene reaction. 2. Prompt versus thermal dissociation of carbonyl oxides to form OH, J. Phys. Chem. A, 2001, 105, 4446-4457.

5 F. Liu, J. M. Beames, A. S. Petit, A. B. McCoy and M. I. Lester, Infrared-driven unimolecular reaction of $\mathrm{CH}_{3} \mathrm{CHOO}$ Criegee intermediates to $\mathrm{OH}$ radical products, Science, 2014, 345, 1596-1598.

6 Y. Fang, F. Liu, V. P. Barber, S. J. Klippenstein, A. B. McCoy and M. I. Lester, Real time observation of unimolecular decay of Criegee intermediates to $\mathrm{OH}$ radical products, J. Chem. Phys., 2016, 144, 061102.

7 R. Atkinson, Gas-phase tropospheric chemistry of volatile organic compounds: 1. Alkanes and alkenes, J. Phys. Chem. Ref. Data, 1997, 26, 215-290.

8 M. E. Jenkin, S. M. Saunders and M. J. Pilling, The tropospheric degradation of volatile organic compounds: a protocol for mechanism development, Atmos. Environ., 1997, 31, 81-104.

9 D. Johnson and G. Marston, The gas-phase ozonolysis of unsaturated volatile organic compounds in the troposphere, Chem. Soc. Rev., 2008, 37, 699-716.

10 D. L. Osborn and C. A. Taatjes, The physical chemistry of Criegee intermediates in the gas phase, Int. Rev. Phys. Chem., 2015, 34, 309-360.

11 T. B. Nguyen, G. S. Tyndall, J. D. Crounse, A. P. Teng, K. H. Bates, R. H. Schwantes, M. M. Coggon, L. Zhang, P. Feiner, D. O. Milller, K. M. Skog, J. C. Rivera-Rios, M. Dorris, K. F. Olson, A. Koss, R. J. Wild, S. S. Brown, A. H. Goldstein, J. A. de Gouw, W. H. Brune, F. N. Keutsch, J. H. Seinfeld and P. O. Wennberg, Atmospheric fates of Criegee intermediates in the ozonolysis of isoprene, Phys. Chem. Chem. Phys., 2016, 18, 10241-10254.

12 O. Welz, J. D. Savee, D. L. Osborn, S. S. Vasu, C. J. Percival, D. E. Shallcross and C. A. Taatjes, Direct kinetic measurements of Criegee intermediate $\left(\mathrm{CH}_{2} \mathrm{OO}\right)$ formed by reaction of $\mathrm{CH}_{2} \mathrm{I}$ with $\mathrm{O}_{2}$, Science, 2012, 335, 204-207.

13 C. A. Taatjes, O. Welz, A. J. Eskola, J. D. Savee, A. M. Scheer, D. E. Shallcross, B. Rotavera, E. P. F. Lee, J. M. Dyke, D. K. W. Mok, D. L. Osborn and C. J. Percival, Direct measurements of conformer-dependent reactivity of the Criegee intermediate $\mathrm{CH}_{3} \mathrm{CHOO}$, Science, 2013, 340, 177-180.

14 L. Sheps, A. M. Scully and K. Au, UV absorption probing of the conformer dependent reactivity of a Criegee intermediate $\mathrm{CH}_{3} \mathrm{CHOO}$, Phys. Chem. Chem. Phys., 2014, 16, 26701-26706.

15 T. Berndt, R. Kaethner, J. Voigtländer, F. Stratmann, M. Pfeifle, P. Reichle, M. Sipilä, M. Kulmala and M. Olzmann, Kinetics of the unimolecular reaction of $\mathrm{CH}_{2} \mathrm{OO}$ and the bimolecular reactions with the water monomer, acetaldehyde and acetone under atmospheric conditions, Phys. Chem. Chem. Phys., 2015, 17, 19862-19873.

16 M. C. Smith, C.-H. Chang, W. Chao, L.-C. Lin, K. Takahashi, K. A. Boering and J. J.-M. Lin, Strong negative temperature dependence of the simplest Criegee intermediate $\mathrm{CH}_{2} \mathrm{OO}$ reaction with water dimer, J. Phys. Chem. Lett., 2015, 6, 2708-2713.

17 W. Chao, J. Hsieh, C. Chang and J. J. Lin, Direct kinetic measurement of the reaction of the simplest Criegee intermediate with water vapor, Science, 2015, 347, 751-754.

18 L. Sheps, B. Rotavera, A. J. Eskola, D. L. Osborn, C. A. Taatjes, K. Au, D. E. Shallcross, M. A. H. Khan and C. J. Percival, The reaction of Criegee intermediate $\mathrm{CH}_{2} \mathrm{OO}$ with water dimer: primary products and atmospheric impact, Phys. Chem. Chem. Phys., 2017, 19, 21970-21979.

19 H.-Y. Lin, Y.-H. Huang, X. Wang, J. M. Bowman, Y. Nishimura, H. A. Witek and Y.-P. Lee, Infrared identification of the Criegee 
intermediates syn- and anti- $\mathrm{CH}_{3} \mathrm{CHOO}$, and their distinct conformation-dependent reactivity, Nat. Commun., 2015, 6, 7012 .

20 O. Welz, A. J. Eskola, L. Sheps, B. Rotavera, J. D. Savee, A. M. Scheer, D. L. Osborn, D. Lowe, A. M. Booth, P. Xiao, M. A. H. Khan, C. J. Percival, D. E. Shallcross and C. A. Taatjes, Rate coefficients of $\mathrm{C}_{1}$ and $\mathrm{C}_{2}$ Criegee intermediate reactions with formic and acetic acid near the collision limit: direct kinetics measurements and atmospheric implications, Angew. Chem., Int. Ed., 2014, 53, 4547-4550.

21 R. Chhantyal-Pun, B. Rotavera, M. R. McGillen, M. A. H. Khan, A. J. Eskola, R. L. Caravan, L. Blacker, D. P. Tew, D. L. Osborn, C. J. Percival, C. A. Taatjes, D. E. Shallcross and A. J. Orr-Ewing, Criegee intermediate reactions with carboxylic acids: a potential source of secondary organic aerosol in the atmosphere, ACS Earth Space Chem., 2018, 2, 833-842.

22 J. M. Beames, F. Liu, L. Lu and M. I. Lester, Ultraviolet spectrum and photochemistry of the simplest Criegee intermediate $\mathrm{CH}_{2} \mathrm{OO}, \mathrm{J}$. Am. Chem. Soc., 2012, 134, 20045-20048.

23 J. M. Beames, F. Liu, L. Lu and M. I. Lester, UV spectroscopic characterization of an alkyl substituted Criegee intermediate $\mathrm{CH}_{3} \mathrm{CHOO}$, J. Chem. Phys., 2013, 138, 244307.

24 F. Liu, J. M. Beames, A. M. Green and M. I. Lester, UV spectroscopic characterization of dimethyl- and ethyl-substituted carbonyl oxides, J. Phys. Chem. A, 2014, 118, 2298-2306.

25 Y.-T. Su, Y.-H. Huang, H. A. Witek and Y.-P. Lee, Infrared absorption spectrum of the simplest Criegee intermediate $\mathrm{CH}_{2} \mathrm{OO}$, Science, 2013, 340, 174-176.

26 Y.-Y. Wang, C.-Y. Chung and Y.-P. Lee, Infrared spectral identification of the Criegee intermediate $\left(\mathrm{CH}_{3}\right)_{2} \mathrm{COO}$, J. Chem. Phys., 2016, 145, 154303.

27 M. Nakajima and Y. Endo, Communication: Determination of the molecular structure of the simplest Criegee intermediate $\mathrm{CH}_{2} \mathrm{OO}$, J. Chem. Phys., 2013, 139, 101103.

28 M. C. McCarthy, L. Cheng, K. N. Crabtree, O. Martinez, T. L. Nguyen, C. C. Womack and J. F. Stanton, The simplest Criegee intermediate $\left(\mathrm{H}_{2} \mathrm{C}=\mathrm{O}-\mathrm{O}\right)$ : isotopic spectroscopy, equilibrium structure, and possible formation from atmospheric lightning, J. Phys. Chem. Lett., 2013, 4, 4133-4139.

29 A. M. Daly, B. J. Drouin and S. Yu, Submillimeter measurements of the Criegee intermediate $\mathrm{CH}_{2} \mathrm{OO}$, in the gas phase, J. Mol. Spectrosc., 2014, 297, 16-20.

30 M. Nakajima and Y. Endo, Spectroscopic characterization of an alkyl substituted Criegee intermediate syn- $\mathrm{CH}_{3} \mathrm{CHOO}$ through pure rotational transitions, J. Chem. Phys., 2014, 140, 011101.

31 M. Nakajima, Q. Yue and Y. Endo, Fourier-transform microwave spectroscopy of an alkyl substituted Criegee intermediate anti-CH ${ }_{3} \mathrm{CHOO}$, J. Mol. Spectrosc., 2015, 310, 109-112.

32 M. Nakajima and Y. Endo, Fourier-transform microwave spectroscopy of dimethyl-substituted Criegee intermediate $\left(\mathrm{CH}_{3}\right)_{2} \mathrm{COO}$, J. Chem. Phys., 2016, 145, 244307.

33 C. Cabezas, J.-C. Guillemin and Y. Endo, Conformational analysis of ethyl-substituted Criegee intermediate by FTMW spectroscopy, J. Chem. Phys., 2016, 145, 224314.
34 C. Cabezas, J.-C. Guillemin and Y. Endo, Conformational preferences of Criegee intermediates: iso-propyl substituted carbonyl oxide, J. Chem. Phys., 2018, 149, 084309.

35 C. Cabezas, J.-C. Guillemin and Y. Endo, Probing the conformational behavior of the doubly substituted methyl-ethyl Criegee intermediate by FTMW spectroscopy, J. Chem. Phys., 2017, 146, 174304.

36 C. Cabezas, I. Kleiner, M. Carvajal and Y. Endo, Probing the methyl torsional barriers of the doubly substituted methylethyl Criegee intermediate by FTMW spectroscopy, J. Mol. Spectrosc., 2018, 353, 23-27.

37 C. Cabezas, J.-C. Guillemin and Y. Endo, Fourier transform microwave spectroscopy of Criegee intermediates: the conformational behaviour of butyraldehyde oxide, J. Chem. Phys., 2019, 150, 104301.

38 C. Cabezas, J.-C. Guillemin and Y. Endo, Fourier-transform microwave spectroscopy of a halogen substituted Criegee intermediate ClCHOO, J. Chem. Phys., 2016, 145, 184304.

39 M. Nakajima and Y. Endo, Spectroscopic characterization of the complex between water and the simplest Criegee intermediate $\mathrm{CH}_{2} \mathrm{OO}$, J. Chem. Phys., 2014, 140, 134302.

40 M. Nakajima and Y. Endo, Observation of hydroxymethyl hydroperoxide in a reaction system containing $\mathrm{CH}_{2} \mathrm{OO}$ and water vapor through pure rotational spectroscopy, J. Chem. Phys., 2015, 143, 164307.

41 C. Cabezas and Y. Endo, The reactivity of the Criegee intermediate $\mathrm{CH}_{3} \mathrm{CHOO}$ with water probed by FTMW spectroscopy, J. Chem. Phys., 2018, 148, 014308.

42 C. Cabezas and Y. Endo, Spectroscopic characterization of the reaction products between Criegee intermediate $\mathrm{CH}_{2} \mathrm{OO}$ and HCl, ChemPhysChem, 2017, 18, 1860-1863.

43 C. Cabezas and Y. Endo, The reaction between the methyl Criegee intermediate and hydrogen chloride: an FTMW spectroscopic study, Phys. Chem. Chem. Phys., 2018, 20, 22569-22575.

44 C. Cabezas and Y. Endo, The Criegee intermediate-formic acid reaction explored by rotational spectroscopy, Phys. Chem. Chem. Phys., 2019, 21, 18059-18064.

45 F. Liu, Y. Fang, M. Kumar, W. H. Thompson and M. I. Lester, Direct observation of vinyl hydroperoxide, Phys. Chem. Chem. Phys., 2015, 17, 20490-20494.

46 H.-J. Werner, P. J. Knowles, G. Knizia, F. R. Manby, M. Schütz, P. Celani, W. Györffy, D. Kats, T. Korona, R. Lindh, A. Mitrushenkov, G. Rauhut, K. R. Shamasundar, T. B. Adler, R. D. Amos, S. J. Bennie, A. Bernhardsson, A. Berning, D. L. Cooper, M. J. O. Deegan, A. J. Dobbyn, F. Eckert, E. Goll, C. Hampel, A. Hesselmann, G. Hetzer, T. Hrenar, G. Jansen, C. Köppl, S. J. R. Lee, Y. Liu, A. W. Lloyd, Q. Ma, R. A. Mata, A. J. May, S. J. McNicholas, W. Meyer, T. F. Miller III, M. E. Mura, A. Nicklaß, D. P. O’Neill, P. Palmieri, D. Peng, K. Pflüger, R. Pitzer, M. Reiher, T. Shiozaki, H. Stoll, A. J. Stone, R. Tarroni, T. Thorsteinsson, M. Wang and M. Welborn, MOLPRO, version 2018.1, 2018, see http://www.molpro.net.

47 T. H. Dunning, Gaussian basis sets for use in correlated molecular calculations. I. The atoms boron through neon and hydrogen, J. Chem. Phys., 1989, 90, 1007-1023. 
48 B. Long, J.-R. Cheng, X. Tan and W. Zhang, Theoretical study on the detailed reaction mechanisms of carbonyl oxide with formic acid, THEOCHEM, 2009, 916, 159-167.

49 L. Vereecken, The reaction of Criegee intermediates with acids and enols, Phys. Chem. Chem. Phys., 2017, 19, 28630-28640.

50 M. Kumar, D. H. Busch, B. Subramaniam and W. H. Thompson, Barrierless tautomerization of Criegee intermediates via acid catalysis, Phys. Chem. Chem. Phys., 2014, 16, 22968-22973.

51 Y. Hirahara, Y. Ohshima and Y. Endo, Pulsed-dischargenozzle Fourier-transform microwave spectroscopy of HC3S $\left({ }^{2} \Pi_{\mathrm{r}}\right)$ and $\mathrm{HC}_{4} \mathrm{~S}\left({ }^{2} \Pi_{\mathrm{i}}\right)$, J. Chem. Phys., 1994, 101, 7342.
52 Y. Endo, H. Kohguchi and Y. Ohshima, PDN-FTMW spectroscopy of open-shell complexes, Faraday Discuss., 1994, 97, 341-350.

53 T. J. Balle and W. H. Flygare, Fabry-Perot cavity pulsed Fourier transform microwave spectrometer with a pulsed nozzle particle source, Rev. Sci. Instrum., 1981, 52, 33.

54 J. K. G. Watson, Vibrational Spectra and Structure, ed. J. R. Durig, Elsevier, Amsterdam, 1977, vol. 6, pp. 1-89.

55 W. Gordy and R. L. Cook, Microwave Molecular Spectra, John Wiley Sons, New York, 1984.

56 R. S. Ruoff, T. D. Klots, T. Emilsson and H. S. Gutowsky, Relaxation of conformers and isomers in seeded supersonic jets of inert gases, J. Chem. Phys., 1990, 93, 3142. 